\title{
An alternative therapy for post-traumatic endophthalmitis: Preserving capsule and primary intraocular lens implantation combined complete vitrectomy
}

\author{
Zhang Zheng*, Chen Min, Wang Qiwei, Ma Jin, Yin Jinfu and Yao Ke \\ Eye Center, Second Affiliated Hospital, School of Medicine, Zhejiang University, Hangzhou, P.R. China.
}

Accepted 30 January, 2012

\begin{abstract}
The purpose of the present study was to investigate whether preserving capsule and primary intraocular lens implantation in vitrectomy could influence the therapeutic effect and safety in the treatment of post-traumatic endophthalmitis. A prospective, randomized, controlled trial was performed in this study. A total of 89 patients with post-traumatic endophthalmitis that occurred between January 2008 and December 2010 were randomly divided into two groups: 45 patients underwent complete vitrectomy with preserved capsule and primary intraocular lens implantation, and 44 patients underwent complete vitrectomy with lensectomy and without intraocular lens implantation. There were no significant differences in patients' age, sex, injuries in detail, intraocular tamponade, culture-proven bacteria, and preoperative and postoperative visual acuity between two groups. No recurrence of bacterial infection was found in any case in both groups, and there was no severe complications happened in two groups during follow-up. Our results suggested that preserving capsule and primary intraocular lens implantation with complete vitrectomy in post-traumatic endophthalmitis was a safe and feasible surgery procedure with benefits in postoperative visual quality, which could be recommended as an alternative therapy.
\end{abstract}

Key words: Post-traumatic endophthalmitis, intraocular lens implantation, capsule, vitrectomy, infection.

\section{INTRODUCTION}

Post-traumatic infectious endophthalmitis is an uncommon but devastating complication of penetrating ocular injuries. It was reported by the United States Eye Injury Registry that $3.4 \%$ cases of open globe injuries are associated with endophthalmitis (Danis, 2002). In these decades, the initial therapy of post-traumatic endophthalmitis is intravitreal and systemic antibiotics administration. When the initial therapy failed to improve the media clarity or visual acuity, the standard treatment is vitrectomy (Novosad et al., 2010; Azad et al., 2003; Essex et al., 2004). Generally, lensectomy was carried

*Corresponding author. E-mail: zhangzhengzju@yahoo.com.cn. Tel: 86-571-87783897. Fax: 86-571-87783897. out prior to vitrectomy in cases with lens rupture or lens opactiy (Azad et al., 2003; Essex et al., 2004; Lekse et al., 2006; Kaushik et al., 2001). During the past, preserving capsule and primary intraocular lens (IOL) implantation in endophthalmitis surgery were not recommended by most surgeons (Essex et al., 2004). A large retrospective study has shown that ruptured lens capsule and IOL were independently associated with the development of post-traumatic endophthalmitis as the other risk factors, such as dirty wound and delay in primary repair (Andreoli et al., 2009). However, with the progress of surgical techniques (e.g. complete vitrectomy instead of core vitrectomy, and silicone oil endotamponade), the chance of successful surgery in endophthalmitis has increased significantly (Azad et al., 2003). Obviously, primary IOL implantation would benefit 
the visual quality of postoperative endophthalmitis patients with aphakia and hyperopia, and there was no new evidence proving that preserving capsule and primary IOL implantation would influence eliminating bacteria and recurrence of endophthalmitis, and increase the incidence of other complications.

Therefore, we conducted this study to compare the recurrence of bacterial infection and other postoperative results of preserving capsule and primary IOL implantation with conventional lensectomy in a prospective randomized controlled trial of patients with post-traumatic endophthalmitis undergoing vitrectomy.

\section{MATERIALS AND METHODS}

\section{Patient selection}

From January 2008 to December 2010, 89 patients (89 eligible eyes) diagnosed with post-traumatic endophthalmitis with lens opactiy at the second affiliated hospital of Zhejiang University were enrolled in this study. There were 68 men (68 eyes) and 21 women (21 eyes), with an average age of 36.8 years (range, $14-79$ years).

A standardized data sheet was completed for each case of endophthalmitis. Information was collected including patient's age, gender, medical and ophthalmic history, and previous vision, if known. The nature of the globe injury was characterized in detail. Any rupture of lens including breach of anterior and posterior lens capsule, nucleus hardness of cataract, intraocular foreign body (IOFB), retinal break and retinal detachment were recorded. Other information including culture results of isolated microorganism, surgical method, use of topical, systemic and intravitreal antibiotics, initial visual acuity and final visual outcomes was also recorded.

The following criteria were used to define the patients:

1. Inclusion criteria were: age $\geq 14$ years; an eye with traumatic history; traumatic cataract with or without lens rupture; presence of typical symptoms (extreme pain, and decreased visual acuity occurring with ocular trauma); presence of typical signs (hypopyon or fibrinoid response and vitritis or vitreous opacification); cornea clear enough for vitrectomy; visual acuity less than 20/200.

2. Exclusion criteria were: fungal endophthalmitis; endogenous endophthalmitis; patients lost to follow up.

All patients underwent emergency surgery within $6 \mathrm{~h}$ after arrival at our hospital. The globe wounds were sutured if occurred. Then intravitreal antibiotics were given (vancomycin $1 \mathrm{mg}$ in $0.1 \mathrm{ml}$ and ceftazidime $2.25 \mathrm{mg}$ in $0.1 \mathrm{ml}$ ) at presentation after aspirating the hypopyon or fibrinoid membrane in anterior chamber. Patients were assessed $24 \mathrm{~h}$ later. All patients who did not show evidence of improvement in media clarity or visual acuity underwent vitrectomy. Vitreous samples were taken at the time of intravitreal injection and vitrectomy, and sent for bacterial and fungal smear, culture and sensitivity testing.

Patients were randomized into two groups: 45 eyes into the IOL implantation group, in which capsule was preserved and IOL was implanted into the intact capsule or ciliary sulcus; 44 eyes into the lensectomy group, in which the lens was cut off completely and no IOL was implanted.

\section{Surgical procedure}

All patients were operated under retrobulbar anesthesia by a single experienced surgeon (Zheng Zhang). In the IOL implantation group, the lens was emulsified and aspirated by phacoemulsification, and
IOL (AMO acrylic, Sensar AR40, USA) was implanted in the capsule in cases without breach of posterior lens capsule; in cases with breach of posterior lens capsule, the anterior lens capsule was preserved as much as possible, and IOL was implanted into ciliary sulcus. In the lensectomy group, the opaque lens and the capsule were completely removed. A complete pars plana vitrectomy was performed and IOFB was taken out by forceps if occured. Then endolaser of any visible or suspicious retinal breaks and $360^{\circ}$ of the peripheral retina near ora serrata was carried out. The silicone oil or gas tamponade were used according to the condition of retinal detachment. Gas tamponade was stilled used in the cases without retinal detachment, if obvious or suspicious retinal breaks could be found.

\section{Systemic and topical medications}

The irrigating solution used in surgery contained vancomycin with concentration of $20 \mathrm{mg} / \mathrm{L}$ as described in previous research (Adenis et al., 1997). No intravitreal antibiotics were injected at the conclusion of the vitrectomy in any patient in either group. Postoperatively, all the patients were continued on intravenous antibiotics (levofloxacin $500 \mathrm{mg} /$ day) for 3 days and intravenous steroids equivalent to prednisolone $1.5 \mathrm{mg} / \mathrm{kg} /$ day for 5 days. Each patient received topical antibiotics (levofloxacin 0.5\%) and topical steroids (prednisolone acetate $1 \%$ ) hourly and cycloplegics (homatropine hydrobromide 2\%) every $4 \mathrm{~h}$. Administration of oral steroids (prednisolone $1.5 \mathrm{mg} / \mathrm{kg}$ ) followed intravenous therapy for another 7 days. The patients were followed up 1, 2, 4 weeks postoperatively and every month in the next 5 months.

\section{Clinical evaluation}

Preoperatively and postoperatively, all patients underwent complete ocular examination including the best corrected visual acuity (BCVA) by Snellen' testing, intraocular pressure measurement, slitlamp biomicroscopic examination of anterior segment and fundoscopy examination. In the cases with silicone oil tamponade, the silicone oil was removed 3 months posteroperatively.

\section{Statistical analysis}

Statistical analysis was performed using SPSS for Windows software (version 16.0, SPSS, Inc.). The Wilcoxon signed ranks test or Chi-square test was used for statistical analysis. $P$ value less than 0.05 was considered statistically significant.

\section{RESULTS}

The characteristics of the patients involved in the study are given in Table 1. The distribution of sex and age was comparable in the two groups $(P=0.660 ; P=0.805)$. The information of detailed injuries of eyes are given in Table 2 , and there were no difference in IOFB, lens rupture, nucleus hardness of cataract and intraoperative retinal breaks between two groups $(P=0.504 ; P=0.394 ; P=0$. $0.843 ; P=0.806)$. The choice of intraocular tamponade was given in Table 2, and there were no significant difference between the two groups $(P=0.562)$.

Bacteria were isolated in $70.8 \%$ of patients $(63 / 89)$. The most common bacterial strain was Staphylococcus epidermidis, which was noted in $47.6 \%$ of the culture- 
Table 1. Patient's characteristics.

\begin{tabular}{lccc}
\hline Characteristics & IOL & LET & $P$ value \\
\hline Case number $(\mathrm{n})$ & 45 & 44 & \\
Sex & & & 0.660 \\
Male $(\mathrm{n})$ & 33 & 35 & \\
Female $(\mathrm{n})$ & 12 & 9 & \\
Age (year) & $36.6 \pm 13.7(14-76)$ & $37.0 \pm 13.2(14-72)$ & 0.805 \\
\hline
\end{tabular}

The difference of sex between two groups was analyzed by Wilcoxon signed ranks test; the difference of age between two groups was analyzed by Chi-square test.

$\mathrm{IOL}=\mathrm{IOL}$ implantation group; LET=lensectomy group.

Table 2. Injuries in detail and intraocular tamponade.

\begin{tabular}{|c|c|c|c|}
\hline Injuries & IOL & LET & $P$ value \\
\hline \multicolumn{4}{|l|}{ Intraocular foreign body } \\
\hline No & 39 & 41 & 0.504 \\
\hline Yes & 6 & 3 & \\
\hline \multicolumn{4}{|l|}{ Lens rupture } \\
\hline No & 7 & 5 & 0.394 \\
\hline Yes & 38 & 39 & \\
\hline Rupture of anterior capsule & 9 & 5 & \\
\hline Rupture of anterior and posterior capsule & 29 & 34 & \\
\hline \multicolumn{4}{|l|}{ Nucleus hardness of cataract } \\
\hline I & 36 & 33 & \\
\hline II & 8 & 10 & 0.843 \\
\hline III & 1 & 1 & \\
\hline \multicolumn{4}{|l|}{ Intraoperative retinal break } \\
\hline No & 8 & 6 & 0.806 \\
\hline Yes & 37 & 38 & \\
\hline \multicolumn{4}{|l|}{ Intraocular tamponade } \\
\hline Saline & 5 & 3 & 0560 \\
\hline Gas & 15 & 19 & 0.562 \\
\hline Silicone oil & 25 & 22 & \\
\hline
\end{tabular}

The data in table 2 were analysed by Chi-square test.

IOL=IOL implantation group; LET=lensectomy group.

positive cases (30/63). Other major bacteria cultured included Staphylococcus aureus in $14.3 \%$ of the cases $(9 / 63)$, and Escherichia coli in $11.1 \%$ of the cases $(7 / 63)$. There was no significant difference in bacterial spectrum between two groups $(P=0.877$, Table 3$)$.

The preoperative and postoperative visual acuity are given in Table 4. BCVA improved significantly both in IOL implantation group and lensectomy group $(P<0.001$; $P<0.001)$. There was no significant difference in visual acuity before and 6 months after surgery between two groups ( $P=0.673, P=0.591)$.

There was no severe intraoperative and postoperative complications happened in two groups, including recurrent bacterial infection, recurrent retinal detachment, expulsive choroidal haemorrhage, chronic hypotony. Silicone oil was removed 3 months postoperatively and the retina remained attached until the last follow-up in all the patients. 
Table 3. Culture-proven bacteria in patients.

\begin{tabular}{|c|c|c|}
\hline Bacteria & IOL $[n(\%)]$ & LET [n(\%)] \\
\hline Culture positive cases & $33(73)$ & $30(68)$ \\
\hline Staphylococcus epidermidis & $16(48.5)$ & $14(46.7)$ \\
\hline Staphylococcus aureus & $4(12.1)$ & $5(16.7)$ \\
\hline Staphylococcus saprophyticus & $1(3.0)$ & $0(0)$ \\
\hline Streptococcus species & $3(9.1)$ & $2(6.7)$ \\
\hline Bacillus subtilis & $1(3.0)$ & $1(3.3)$ \\
\hline Bacillus cereus & $1(3.0)$ & $0(0)$ \\
\hline Bacillus aerogenes capsulatus & $1(3.0)$ & $0(0)$ \\
\hline Microbacterium & $0(0)$ & $1(3.3)$ \\
\hline Escherichia coli & $3(9.1)$ & $4(13.3)$ \\
\hline Pseudomonas aeruginosa & $1(3.0)$ & $1(3.3)$ \\
\hline Pseudomonas spec & $1(3.0)$ & $0(0)$ \\
\hline Bacillus proteus & $1(3.0)$ & $2(6.7)$ \\
\hline$P$ value & & \\
\hline
\end{tabular}

The difference of bacteria spectrum between two groups was analyzed by Chi-square test. $\mathrm{IOL}=\mathrm{IOL}$ implantation group; LET=lensectomy group.

Table 4. Preoperative and posteroperative visual acuity.

\begin{tabular}{|c|c|c|c|c|}
\hline \multirow{2}{*}{ BCVA } & \multicolumn{2}{|c|}{ IOL (n) } & \multicolumn{2}{|c|}{ LET (n) } \\
\hline & pre & post $(6 \mathrm{~m})$ & pre & post $(6 \mathrm{~m})$ \\
\hline $\mathrm{LP} \leq \mathrm{BCVA} \leq \mathrm{HM}$ & 38 & 0 & 40 & 0 \\
\hline $\mathrm{BCVA}=\mathrm{CF}$ & 5 & 13 & 3 & 10 \\
\hline$C F<B C V A \leq 20 / 400$ & 1 & 5 & 1 & 8 \\
\hline $20 / 400<B C V A \leq 20 / 200$ & 1 & 6 & 0 & 10 \\
\hline $20 / 200<B C V A \leq 20 / 60$ & 0 & 15 & 0 & 12 \\
\hline $20 / 60<B C V A \leq 20 / 40$ & 0 & 6 & 0 & 4 \\
\hline \multicolumn{2}{|c|}{$P$ value (preoperative IOL:LET) } & & \multicolumn{2}{|c|}{0.673} \\
\hline \multicolumn{2}{|c|}{$P$ value (postoperative IOL:LET) } & & \multicolumn{2}{|c|}{0.591} \\
\hline
\end{tabular}

The difference of BCVA between two groups was analyzed by Chi-square test. IOL=IOL implantation group; LET=lensectomy group; BCVA= best corrected visual acuity; pre=preoperative; post=postoperative; $6 \mathrm{~m}=\mathrm{six}$ months after surgery; $\mathrm{LP}=$ light perception; $\mathrm{HM}=$ hand movement; $\mathrm{CF}=$ counting fingers.

\section{DISCUSSION}

Endophthalmitis is a destructive complication of penetrating ocular trauma. It is an important cause of visual failure following open globe injuries, so complete removal of intraocular microorganisms, especially in the vitreous cavity, is the most important critical point in treating endophthalmitis and preventing recurrence.

The role of vitrectomy in treating endophthalmitis is very important, because vitrectomy can:

(1) Remove a sizeable portion of the bacteria, associated toxins, and inflammatory cells in the vitreous cavity (Conway et al., 1986);

(2) clear the media by removing opacified and dense vitreous body;
(3) Prevent vitreous tractional bands formation that may cause retinal detachment (Hadden,1981).

The extent of vitrectomy to be performed in endophthalmitis is controversial now. Some surgeons advocated a complete vitrectomy for eliminating bacteria as much as possible (Rahman et al., 2000), but the others advocated a more limited vitrectomy-core vitrectomy due to the risk of creating a retinal break (Bhagat et al., 2011), and causing hemorrhage (Donahue et al., 1993). However, Azad and coworkers, showed successful complete vitrectomy with the help of silicone oil tamponade in treating post-traumatic endophthalmitis (Azad et al., 2003). In their case series, the visual acuity in patients treated with complete vitrectomy and silicone oil tamponade were better than patients treated with core 
vitrectomy. None of patients treated in complete vitrectomy and silicone oil tamponade group developed rhegmatogenous retinal detachment, but the rate of patients in the core vitrectomy group was 33.3\%. Due to the tamponading effect of silicone oil on possible occult retinal tears, more researches demonstrated a good prognosis in post-traumatic endophthalmitis treating with complete vitrectomy and silicone oil tamponade in these years (Yan et al., 2008; Lin et al., 2011).

In our research, treating patients with complete vitrectomy also showed good results. There were no recurrence of endophthalmitis, rhegmatogenous retinal detachment, and other severe complications happened after surgery in both two groups. As the patients treated with complete vitrectomy in Azad's research showed good final visual acuity(better than or equal to 20/200 in 7 of 12 cases (58.3\%)) (Azad et al., 2003), the final BCVA of patients in our research was better than or equal to $20 / 200$ in 37 of 89 cases (41.6\%). Azad attributed better visual outcome in the complete vitrectomy group to less inflammatory chorioretinal damage due to a more complete removal of the microbial load. We believed that performing vitrectomy early was another factor. Antibiotics in vitreous may cause bacteria to lyse and thus may result in release of toxins which will damage retina and chorioid persistently even if the bacteria have been eradicated. Vitrectomy can clear such toxins if performed within 24 to $48 \mathrm{~h}$ after intravitreal antibiotics injections (Verbraeken et al., 1988). We also contributed the good visual outcome to endolaser and proper tamponade on visible and possible occult retinal breaks, which prevented the happening of retinal detachment postoperatively.

Certainly, it might be difficult in performing complete vitrectomy early in post-traumatic endophthalmitis patients due to corneal haziness. However, some skills may help surgeons to overcome the difficulties. We usually used glycerin to dehydrate cornea edema before and in surgery.

Visibility would be better after lens and anterior vitreous body was removed, because there was enough space for circulation of irrigating fluid to remove the inflammatory particles. At that time, the space in anterior chamber and anterior vitreous cavity would become clearer, and corneal haziness would reduce further. When the surgeon removed the posterior hyaloid and gained a view of the retina and optic nerve, the reflect light from posterior pole would help increasing the visibility in surgery. Based on these skills, doing vitrectomy on cases with moderate corneal haziness was not very difficult for a sophisticated doctor. However, we do not recommend vitrectomy early in all cases, especially in cases with severe corneal haziness, which should undergo vitrectomy by endoscope.

In past researches, silicone oil tamponade was widely used after complete vitrectomy in treating endophthalmitis (Bali et al., 2003; Wang et al., 2007;
Siqueira et al., 2009; Nashed et al., 2011; Lin et al., 2011). Conventionally, silicone oil tamponaded on possible retinal breaks, and prevented the formation of postoperative proliferative vitreoretinopathy. In addition, it was reported that silicone oil had an antimicrobial activity against $S$. aureus, $S$. epidermidis, $P$. aeruginosa, $C$. albicans, and Aspergillus spp., which are common endophthalmitis-causing agents in vitro. (Ozdamar et al., 1999), and other results in clinical research suggested that silicone oil tamponade might be beneficial in the treatment strategy of infectious endophthalmitis (Yan et al., 2008; Siqueira et al., 2009). Empirically, we did not use silicone oil as antimicrobial agent. But the residual numbers of bacteria was declined significantly after complete vitrectomy and flushing of irrigating solution containing vancomycin, and the living space of bacteria was very limited after silicone oil or gas tamponade. The residual bacteria in very limited vitreous space could be destroyed by leucocytes and intravenous antibiotics easily.

In our research, preserving capsule and primary IOL implantation combined with complete vitrectomy showed good effects. First, there was no recurrence of bacterial endophthalmitis in $\mathrm{IOL}$ implantation group as in lensectomy group, although preserving capsule with very little residual cortex and primary IOL implantation were two important risk factor in the development of posttraumatic endophthalmitis (Andreoli et al., 2009). We considered that complete vitrectomy with gas or silicone oil tamponade provided strong supports for complete removal of bacteria. Second, there was no other severe complications happened in IOL implantation group, despite preserving capsule and primary IOL implantation which would increase difficulty of the surgery, such as decreased visibility in the stage of gas liquid exchange. None of patients developed postoperative retinal detachment in both IOL implantation group and lensectomy group. We believed that endolaser of any visible or suspicious retinal breaks and $360^{\circ}$ of the peripheral retina near ora serrata (examination with indentation), and silicone oil or gas tamponade prevented the happening of postoperative retinal detachment. Third, there was no difference in BCVA between two groups. It is obvious that pseudophakic eyes with low ametropia in IOL implantaion group would have better uncorrected visual acuity, and obtain binocular balance easily when corrected by glasses. However, aphakic eyes with high hyperopia in lensectomy group would have lower uncorrected visual acuity than eyes in IOL implantation group. Although the aphakic eyes might obtain binocular balance by rigid gas permeable contact lens (RGP), they still have the problem that the RGP was not accepted by a considerable proportion of aphakic patients after trauma due to dry eye (Itoh et al., 1999), inconvenience (Ky et al., 1998; McMahon et al., 1997; Ferris, 1979), and difficulty in wearing it caused by corneal scar.

In summary, c ompared to lensectomy with complete 
vitrectomy, preserving capsule and primary IOL implantation with complete vitrectomy in post-traumatic endophthalmitis benefited the postoperative visual acuity and quality, and did not influence the incidence of recurrence of bacterial infection, postoperative retinal detachment, and other severe complications of surgery. So this therapy was safe and feasible, and should be considered a good alternative surgical method. Certainly, a large-scale study including more post-traumatic endophthalmitis cases is still needed in the future, which will be used to seek possible but rare postoperative reinfection cases.

\section{ACKNOWLEDGEMENTS}

The research was supported by Zhejiang Province Key Lab Fund, and Traditional Chinese Medicine Foundation of Zhejiang Province ( 2011ZB078).

\section{REFERENCES}

Adenis JP, Robert PY, Mounier M, Denis F (1997). Anterior chamber concentrations of vancomycin in the irrigating solution at the end of cataract surgery. J. Cataract Refract. Surg., 23(1): 111-4.

Andreoli CM, Andreoli MT, Kloek CE, Ahuero AE, Vavvas D, Durand ML (2009). Low rate of endophthalmitis in a large series of open globe injuries. Am. J. Ophthalmol., 147(4): 601-8.

Azad R, Ravi K, Talwar D, Rajpal, Kumar N (2003). Pars plana vitrectomy with or without silicone oil endotamponade in posttraumatic endophthalmitis. Graefes Arch. Clin. Exp. Ophthalmol., 241(6): 478-83.

Bali E, Huyghe P, Caspers L, Libert J (2003). Vitrectomy and silicone oil in the treatment of acute endophthalmitis. Preliminary results. Bull. Soc. Belge Ophtalmol., (288): 9-14.

Bhagat N, Nagori S, Zarbin M (2011). Post-traumatic Infectious Endophthalmitis. Surv Ophthalmol., 56(3): 214-51.

Conway BP, Campochiaro PA (1986). Macular infarction after endophthalmitis treated with vitrectomy and intravitreal gentamicin. Arch. Ophthalmol. 104(3): 367-71.

Danis RP (2002). Endophthalmitis. Ophthalmol. Clin. North Am., 15(2): 243-8.

Donahue SP, Kowalski RP, Jewart BH, Friberg TR (1993). Vitreous cultures in suspected endophthalmitis. Biopsy or vitrectomy? Ophthalmology, 100(4): 452-5.

Essex RW, Yi Q, Charles PG, Allen PJ (2004). Post-traumatic endophthalmitis. Ophthalmology, 111(11): 2015-22.

Ferris RL (1979). Complications associated with aphakic contact lenses. Ophthalmology, 86(6): 1120-3.

Hadden OB (1981). Vitrectomy in the management of endophthalmitis. Aust. J. Ophthalmol., 9(1): 27-32.

Itoh R, Yokoi N, Kinoshita S (1999). Tear film instability induced by rigid contact lenses. Cornea, 18(4): 440-3.

Kaushik S, Ram J, Dogra MR, Narang S, Brar GS, Gupta A (2001). Traumatic lens abscess with chornic endophthalmitis successfully treated with pars plana lensectomy and vitrectomy. Ophthalmic Surg. Lasers., 32(3): 239-42.

Ky W, Scherick K, Stenson S (1998). Clinical survey of lens care in contact lens patients. CLAO J., 24(4): 216-9.

Lekse Kovach J, Maguluri S, Recchia FM (2006). Subclinical endophthalmitis following a rooster attack. J. AAPOS, 10(6): 579-80.

Lin H, Ling S, Liu Z, Zhong X, Chen W (2011). Preventive scleral buckling and silicone oil tamponade are important for posttraumatic endophthalmitis successfully managed with vitrectomy. Ophthalmologica. 226(4): 214-9.
McMahon TT, Devulapally J, Rosheim KM, Putz JL, Moore M, White S (1997). Contact lens use after corneal trauma. J Am. Optom. Assoc., 68(4): 215-24.

Nashed A, Saikia P, Herrmann WA, Gabel VP, Helbig H, Hillenkamp J (2011). The outcome of early surgical repair with vitrectomy and silicone oil in open-globe injuries with retinal detachment. Am. J. Ophthalmol., 151(3): 522-8.

Novosad BD, Callegan MC (2010). Severe bacterial endophthalmitis: towards improving clinical outcomes. Expert Rev. Ophthalmol., 5(5): 689-698.

Ozdamar A, Aras C, Ozturk R, Akin E, Karacorlu M, Ercikan C (1999). In vitro antimicrobial activity of silicone oil against endophthalmitiscausing agents. Retina., 19(2): 122-6.

Siqueira RC, Gil AD, Canamary F, Minari M, Jorge R (2009). Pars plana vitrectomy and silicone oil tamponade for acute endophthalmitis treatment. Arq. Bras. Oftalmol., 72(1): 28-32.

Rahman MK, Holz ER (2000). Alcaligenes xylosoxidans and Propionibacterium acnes postoperative endophthalmitis in a pseudophakic eye. Am. J. Ophthalmol., 129(6): 813-5.

Verbraeken H, Geeroms B, Karemera A (1988). Treatment of endophthalmitis by pars plana vitrectomy. Ophthalmologica, 197(1): $19-25$.

Wang Z, Dong N, Kang Y, Liu X, Cao Q, Liang D, Lin X (2007).Clinical study of pars plana vitrectomy with silicone oil endotamponade in posttraumatic endophthalmitis on eyes without retinal detachment. Yan Ke Xue Bao., 23(1): 48-52.

Yan H, Lu Y, Yu J, Han J, Zhang J, Chen S, Xu Y (2008). Silicone oil in the surgical treatment of traumatic endophthalmitis. Eur $J$ Ophthalmol.,18(5): 680-4. 